\title{
Osteartrosis y pie. Bases de tratamiento biorregulador
}

\author{
Osteoarthrosis and foot. Basis of bioregulator treatment \\ Debora María García ACedo"***, Fernando Ares Bella* \\ * Diplomado en Podología \\ podología_roma@hotmail.com \\ ** Experto Universitario en Podología Médico-Quirúrgica de Antepie \\ podología_roma@hotmail.com
}

Correspondencia:

Fernando Ares Bella

Podología Roma

Plaza Manuel Becerra 18

28028 Madrid (España)

E-mail: podología_roma@hotmail.com

Fecha de recepción: 9 abril de 2010

Fecha de aceptación: 22 de febrero de 2011

Los autores declaran no tener ningún tipo de interés económico o comercial.

\section{RESUMEN}

La OsteoArtrosis (OA) es un síndrome complejo, que afecta a las estructuras no solo articulares sino periarticulares como ligamentos, tendones y sistema miofascial, generando un cuadro clínico en el que predomina los síntomas neurogénicos, a consecuencia de un fenómeno conocido como "facilitación" o "campo interferente". Las últimas investigaciones avalan como causa primaria de la OA las cargas inadecuadas soportadas por el complejo articular debido al mal alineamiento segmentario; y la inflamación sinovial, antaño causa inicial, como proceso secundario a la presencia de fragmentos cartilaginosos, debido a su deterioro, en el espacio articular. La Biopuntura de medicamentos Biorreguladores aporta una perspectiva terapéutica que abarca todos los elementos implicados en esta patología, su clínica y el proceso degenerativo del "órgano articular".

Palabras clave: osteoartrosis; pie; condrocito; dolor; facilitación.

\begin{abstract}
Osteoarthritis (OA) is a complex syndrome, which affects not only the structures but periarticular joint such as ligaments, tendons and myofascial system, resulting in a clinically predominant neurogenic symptoms as a result of a phenomenon known as "facilitation" or "interference field." Recent investigations support as the primary cause of OA inappropriate burdens borne by the joint complex due to segmental malalignment, and synovial inflammation, once the initial cause, as a process secondary to the presence of cartilage fragments, due to deterioration in the joint space. The drug Biopuntura Bioregulators provides a therapeutic perspective that encompasses all the elements involved in this pathology, clinical history and the degenerative process of "joint authority."
\end{abstract}

Key words: osteoarthritis; foot; condrocyte; pain; facilitation.

Sumario: 1. Introducción, 2. Fisiopatología, 3. Clínica, 4. Diagnóstico, 5. Bases para el tratamiento biorregulador, 6. Conclusiones, Bibliografía.

Referencia normalizada: García Acedo, DM., Ares Bella, F. Osteartrosis y pie. Bases de tratamiento biorregulador Rev. Int. Cienc. Podol. 2013; 7(1): 23-32. 


\section{INTRODUCCIÓN}

Se ha llevado a cabo una búsqueda bibliográfica en las bases de datos de Medline y Pudmed, además de textos especializados, revisando un total de 126 artículos. La OsteoArtrosis es el proceso degenerativo que afecta a articulaciones diartródiales, o lo que es lo mismo, articulaciones sinoviales. Se considera la patología de mayor prevalencia en Reumatología, y una de las de mayor coste socioeconómico en las sociedades occidentales, por el elevado número de incapacidad laboral que produce. Las áreas de afectación más frecuente y mejor estudiada son manos, caderas, rodillas y columna cérvico-lumbar. La referencia a la presencia del deterioro articular en el pie, fundamentalmente en primera AMTF (Figura 1), es escaso y no hemos hallado estudios suficientemente relevantes. Uno de los motivos para que esta falta de interés pensamos que, quizás, sea que la presencia de esta patología en la primera AMFG sea proporcionalmente mayor en personas por encima de los 65 años, por lo que su coste sociolaboral es significativamente menor. Sin embargo, la incapacidad funcional que genera produce alteraciones en los patrones de marcha que a su vez, pueden formar parte de la cadena de génesis y perpetuación de los fenómenos desencadenantes o agravantes de la localización de esta patología en estructuras de carga por encima del pie.

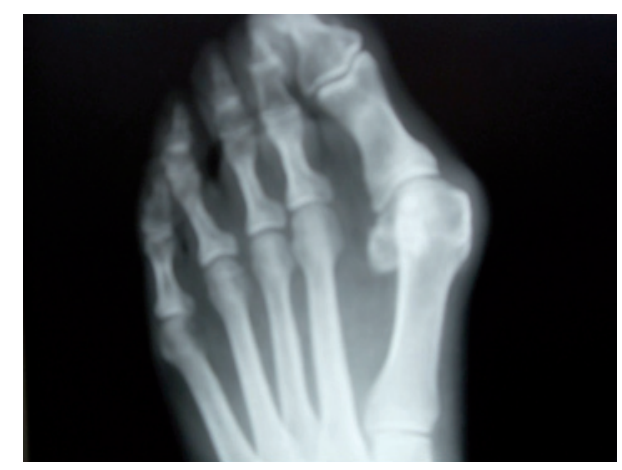

Figura 1. Presencia de signos OA en AMTF.

\section{FISIOPATOLOGÍA}

El signo estructural característico de la OA es la pérdida progresiva del cartílago articular, aumento de espesor de la placa subcondral, neoformación ósea (Figura 2), en los márgenes de la articulación y el desarrollo de quistes óseos subcondrales.

Las investigaciones recientes señalan la OA como un proceso degenerativo articular, originalmente no inflamatorio, cuyo factor desencadenante es la combinación de oscilación y aplicación de peso y carga ${ }^{1}$. El factor principal no es la cantidad de carga sino el mecanismo que la produce ${ }^{2}$. La presencia de sinovitis en el cuadro clínico parece responder al desprendimiento de fragmentos de cartílago deteriorado

Tabla 1. Etiología clásica de la OA.

\section{Causas principales de OA ()}

1. Sobrecarga y carga anómala.

2. Displasias.

3. Posiciones anómalas de las articulaciones y de los ejes de origen congénito o postraumático.

4. Sobrecarga estática (profesión, sobrepeso, deporte).

5. Acontecimientos traumáticos directos.

6. Alteraciones estructurales de las partes óseas de la articulación (necrosis óseas asépticas, ostecondrosis disecante, enfermedad de Paget, etc.)

7. Enfermedades metabólicas (gota, condrocalcinosis, etc).

8. Procesos inflamatorios. 


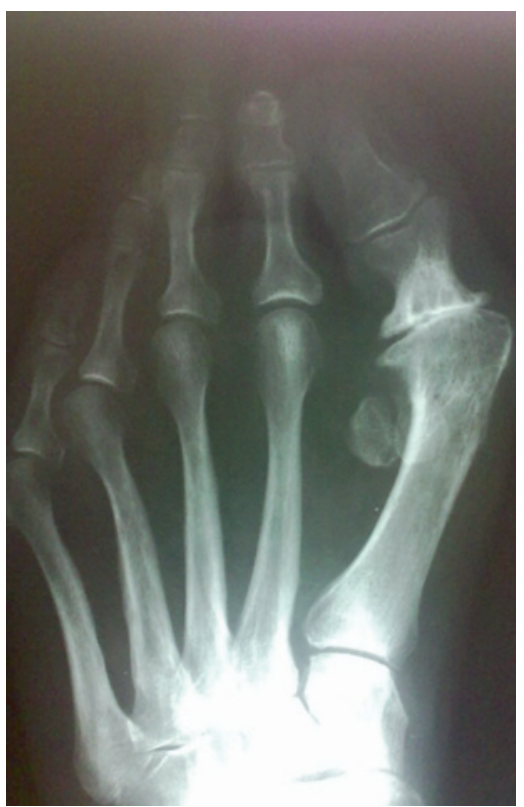

Figura 2. Neoformación ósea en la periferia del cartílago articular de $1^{\mathrm{a}}$ art. MTFG.

en la cavidad $\operatorname{articular}^{3}$, producidos por el estrés articular y favorecido por factores desencadenantes o agravantes Tabla 1, como el deterioro de las fibras de colágeno, debido, por ejemplo, a la edad. Diferentes hipótesis han atribuido a este proceso la alteración del metabolismo del condrocito, la angiogénesis y el aumento de la regeneración ósea ${ }^{4}$. La sinovitis crónica se asocia con alteración de los nervios sensitivos locales y cambios en la síntesis y liberación de neurotransmisores y neuromoduladores ${ }^{7}$. Se produce la hiperactividad celular en la sinovial inflamada: fibroblastos y proliferación de vasos sanguíneos, reclutamiento de macrófagos y aumenta la apoptosis celular ${ }^{8}$. El hecho es que la inflamación no es, a diferencia de las patologías inflamatorias articulares, como la artritis reumatoide o la espondiloartrosis seronegativa, un factor principal sino secundario, lo que modifica la perspectiva terapéutica. El desequilibrio de la actividad metabólica de los condrocitos va a desencadenar un ciclo de hiperactividad anabólica/catabólica (la activación de vía de señalización catabólica e inhibición de vías anabólicas) a consecuencia del estrés: la liberación de mediadores inflamatorios, citocinas y productos de degradación de la matriz que estimulan las metaloproteinasas con el objetivo de inducir la degradación de los tejidos lesionados, contrarrestados por factores de crecimiento, principalmente los TGF- $\beta$, y proteínas morfogénicas óseas con el fin de reparar el tejido. La pérdida de estos últimos o la incapacidad para responder ante ellos, como ocurre en la vejez, desencadena este proceso degenerativo ${ }^{10}$. Otro de los elementos implicados en la destrucción articular es la liberación de Radicales Libres (ROS, reactyve oxygen species), particularmente, la combinación de óxido nítrico y otros ROS para formar el altamente tóxico, peroxinitrito. Los ROS inducen moduladores inflamatorios, como NF-kB, IL-1, IL-6, inducen la apoptosis del condrocito e intervienen en la producción de VEGF, factor de crecimiento del endotelio vascular, implicado en la angiogenia y la inflamación crónica ${ }^{9}$. Este fenómeno explica la formación osteofitaria, por la osificación osteocondral. La angiogenia, además, genera neoinervación, lo que puede explicar en parte el cuadro doloroso.

\section{CLÍNICA}

La clínica de la OA en la primera AMTF, la de aparición más frecuente en el pie, se caracteriza por el dolor de tipo mecánico, rigidez/bloqueo articular y un grado elevado de hipersensibilidad en el área afectada. La consecuencia inmediata es el cambio en los patrones de carga $\mathrm{y}$, por tanto, la alteración de la biomecánica, alejándonos de los patrones de normalidad por compensación segmentaria (Figura 3). Esta desviación de la normalidad comporta el malfuncionamiento muscular y de las estructuras

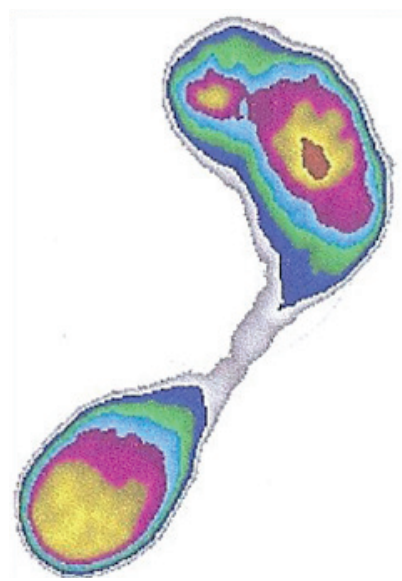

Figura 3. Imagen podobarométrica de transferencia de carga por Hallux Limitus. 
de soporte, en un intento de protección neuromuscular, como tendones y ligamentos, que contribuyen a la alteración de la propiocepción $y$, en zona de fascia, a la activación de nociceptores y la transmisión del dolor. Este cuadro disfuncional óseo-periarticular actúa de factor perpetuante de la OA.

Durante el examen físico podemos detectar los siguientes signos ${ }^{5}$ :

- Ensanchamiento óseo.

- Inestabilidad articular

- Crepitaciones óseas.

- Inestabilidad y limitación de los movimientos.

\section{DIAGNÓSTICO}

\section{Bioquímica}

Dado el carácter insidioso en el inicio de la OA, uno de los objetivos de la investigación es localizar marcadores biológicos fiables que nos orientaran de la presencia de este proceso degenerativo. Se han descrito un número de marcadores ligados a la inflamación sinovial como el COMP (cartilage oligomeric protein) y los niveles séricos de Proteina C-reactiva (CRP) y ácido Hialurónico (HA), como factores de riesgo para el desarrollo de la $\mathrm{OA}^{6}$.

\section{Diagnóstico por imagen}

En imagen radiográfica la alteración degenerativa de la primera AMTF del pie se caracteriza por estrechamiento del espacio articular, formación osteofitaria, esclerosis y formación de quistes subcondrales ${ }^{7,8}$ (Figura 4).

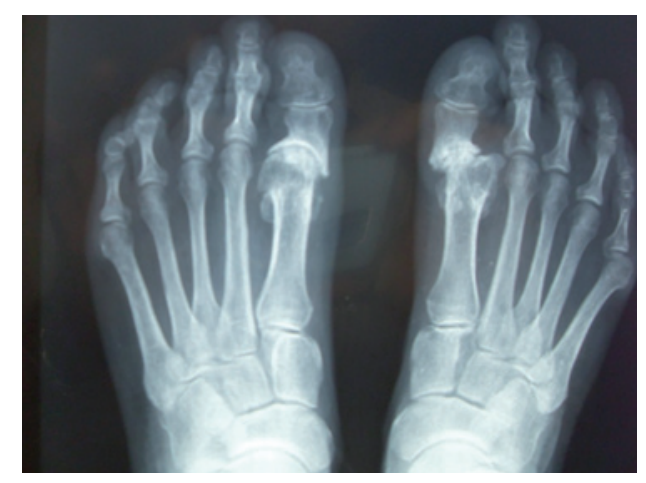

Figura 4. Signos típicos de la OA en primera AMTF en paciente post quirúrgico.
La RMN es una de las pruebas diagnósticas emergentes gracias a la precisión y a la cantidad de datos que nos puede aportar como la morfología del cartílago, su composición, la presencia de líneas de fisura, desprendimiento de tejido cartilaginoso en la cavidad articular, la presencia de sinovitis o la situación de la estructura articular (ligamentos, capsula articular, tendones), la estructura y composición ósea, etc. Por sus características, este tipo de imagen es la mejor opción diagnóstica tanto para la investigación como para la clínica e inserción de protocolos de tratamiento ${ }^{9,10}$.

\section{BASES PARA EL TRATAMIENTO BIORREGULADOR}

Dados los avances en la comprensión de la génesis y evolución de la OA, la terapéutica actual basada en analgésicos y antiinflamatorios no esteroideo parece abocada al fracaso. La no presencia de células inflamatorias e infiltrado linfocitario, y, debido a la baja vascularización del cartílago articular, la escasa permeabilidad del mismo hacen muy difícil que el efecto de las fármacos realicen ninguna función localizada en el área afectada. Este hecho sumado a los diversos efectos adversos, irritación de mucosa gastrointestinal, lesiones de la mucosa, ulceración y hemorragias, así como otro tipo de alteraciones como los recientemente descritos riesgos cardiovasculares de los antiinflamatorios específicos de la COX-2 ${ }^{18}$, en una población principalmente de edad avanzada, con el consiguiente posible deterioro de los sistemas de detoxificación y drenaje (hígado, riñones y sistema linfático) hacen aconsejable otras perspectivas terapéuticas. Así pues debemos planificar otro tipo de abordaje terapéutico basado en la estimulación de las vías fisiológicas de respuesta.

Según los principios epistemológicos de la Medicina Biorreguladora lo que entendemos por "Enfermedad" es la manifestación de la lucha de los Sistemas de Regulación para eliminar el elemento desequilibrante de la Homeodinamia.

Básicamente, la patología en el organismo evoluciona en tres planos, el humoral, sobre el que interactúan los sistemas neuroendocrino e 


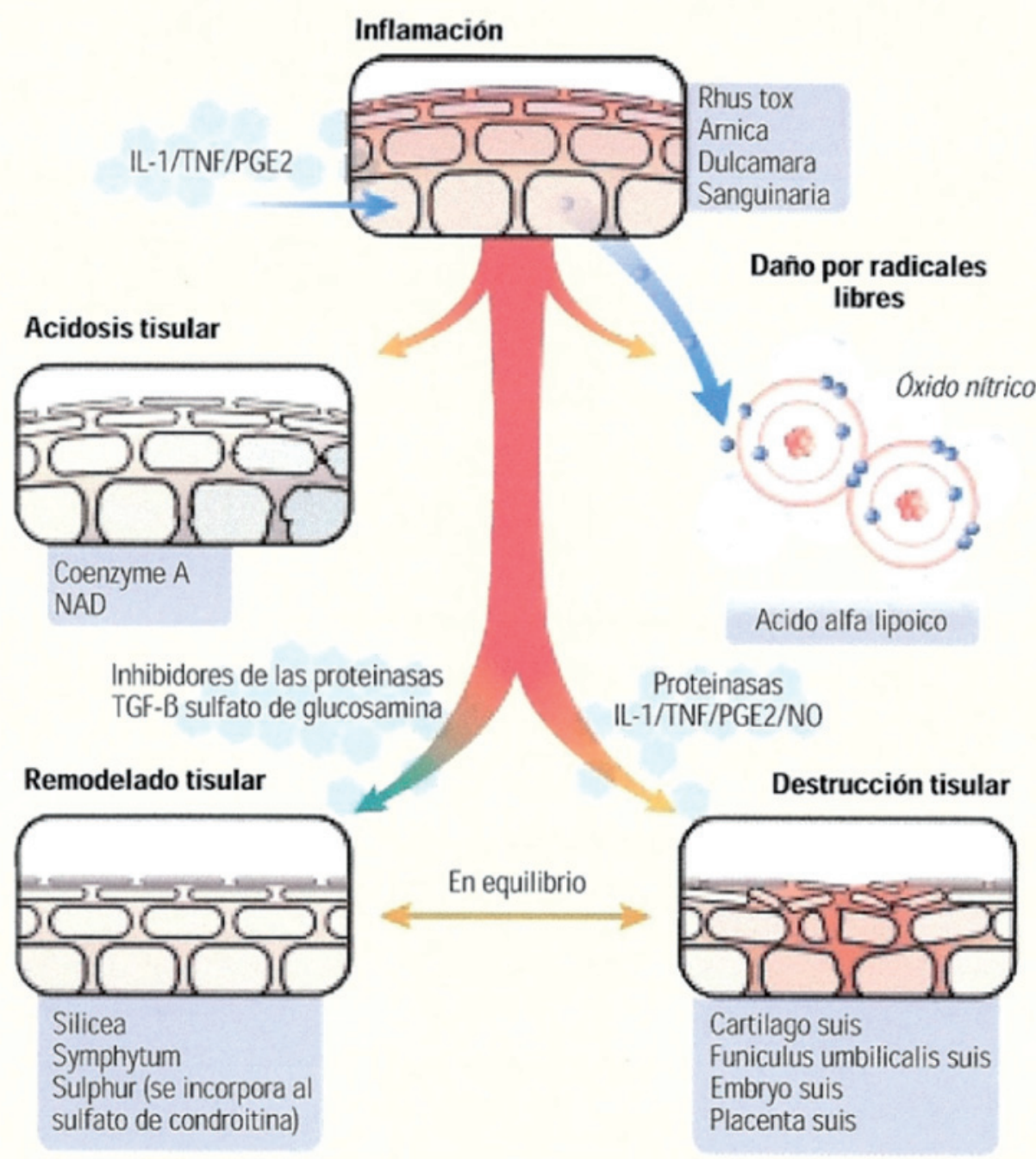

Figura 5. Principios activos del Medicamento ZH y su tropismo funcional sobre el proceso de destrucción del cartílago. Reprinted with permission from Medicina Biológica. 2004;17(4):58.

inmunológico, y que desarrollan respuestas de tipo excretoras, por las vías fisiológicas, y la respuesta inflamatoria. El espacio extracelular, la matrix (MEC), donde se produce la deposición de toxinas y la impregnación de la membrana celular $^{11}$. Es el inicio de la cronicidad y la alteración funcional y metabólica de los sistemas afectados. Por último, se produce la invasión celular, la desnaturalización tisular en forma de degeneración y desdiferenciación. La clasificación de la OA nos facilita la comprensión de la evolución de la patología, los sistemas implicados y la planificación de la terapéutica biorreguladora (Tabla 2).

\section{Medicamento biorregulador}

El Medicamento Biorregulador "es un producto médico que no suprime las reacciones neuroinmunológicas ni interfiere con los sistemas fisiológicos de defensa del organismo"19, que nos permite abarcar todas las facetas que presenta una patología compleja como la OA. Los componentes de estos medicamentos cumplen dos características: organotropismo, es decir 
Tabla 2. Tabla de Evolución de las Enfermedades aplicada a la patología musculoesquelética y su tratamiento con medicamentos Bioreguladora. Reprinted with permission from Medicina Biológica. 2004;17(4):58.

\begin{tabular}{|c|c|c|c|c|c|c|c|}
\hline \multirow[b]{2}{*}{$\begin{array}{l}\text { Sistema } \\
\text { orgánico }\end{array}$} & \multicolumn{2}{|c|}{ FASES HUMORALES } & \multicolumn{3}{|c|}{ FASES DE MATRIZ } & \multicolumn{2}{|c|}{ FASES CELULARES } \\
\hline & $\begin{array}{l}\text { Fases de } \\
\text { excreción }\end{array}$ & $\begin{array}{l}\text { Fases de } \\
\text { inflamación }\end{array}$ & $\begin{array}{l}\text { Fases de } \\
\text { deposición }\end{array}$ & & $\begin{array}{l}\text { Fases de } \\
\text { inpregnación }\end{array}$ & $\begin{array}{l}\text { Fases de } \\
\text { degeneración }\end{array}$ & $\begin{array}{l}\text { Fases de des- } \\
\text { diferenciación }\end{array}$ \\
\hline \multirow{3}{*}{$\begin{array}{l}\text { Aplicación } \\
\text { de grupos } \\
\text { terapéuticos } \\
\text { conforme a la } \\
\text { Tabla de las Seis } \\
\text { Fases y sus } \\
\text { tratamientos }\end{array}$} & $\begin{array}{r}\text { Patolog } \\
\text { Medicamentos comf } \\
\text { y min }\end{array}$ & $\begin{array}{l}\text { aguda } \\
\text { uestos de vegetales } \\
\text { rales }\end{array}$ & & & & . & \\
\hline & & \multicolumn{4}{|c|}{$\begin{array}{l}\text { Patología funcional } \\
\text { Homaccords, medicamentos compuestos de vegetales } \\
\text { y minerales }\end{array}$} & & \\
\hline & & & \multicolumn{4}{|c|}{$\begin{array}{l}\text { Patología crónico-degenerativa } \\
\text { Organopreparados-"suis", nosodes, compositum }\end{array}$} & \\
\hline Piel & Crisis sudorales & Acné & Nevos & \multirow{5}{*}{$\begin{array}{l}\text { 엉 } \\
\text { 응 } \\
\text { 음 } \\
\text { 뜸 } \\
\text { 응 } \\
\end{array}$} & Alergia & Esclerodermia & Melanoma \\
\hline $\begin{array}{l}\text { Sistema } \\
\text { sensorial }\end{array}$ & Lagrimeo, ottorrea & $\begin{array}{l}\text { Conjuntivitis, } \\
\text { otitis media }\end{array}$ & $\begin{array}{l}\text { Calacio, } \\
\text { colesteatoma }\end{array}$ & & $\begin{array}{l}\text { Iridociclitis, } \\
\text { acufenos }\end{array}$ & $\begin{array}{l}\text { Degeneración } \\
\text { macular, anosmia }\end{array}$ & $\begin{array}{l}\text { Amaurosis, tumor } \\
\text { maligno }\end{array}$ \\
\hline \multirow[t]{2}{*}{$\begin{array}{l}\text { Sistema } \\
\text { locomotor }\end{array}$} & Artralgias & Epicondilitis & Exostosis & & $\begin{array}{l}\text { Artritis reumatoide } \\
\text { cronica }\end{array}$ & $\begin{array}{l}\text { Espondilosis, } \\
\text { osteoartritis }\end{array}$ & $\begin{array}{l}\text { Osteosarcoma, } \\
\text { condroma }\end{array}$ \\
\hline & Mialgia & Esguince agudo & Miogelosis & & Fibromialgia & Distrofia muscular & Miosarcoma \\
\hline $\begin{array}{l}\text { Enfermedad } \\
\text { musculo- } \\
\text { esquelética }\end{array}$ & Artralgia & $\begin{array}{l}\text { Esguinces agudos, } \\
\text { reacciones } \\
\text { inflamatorias } \\
\text { agudas de la } \\
\text { cápsula articular، } \\
\text { tendones y } \\
\text { ligamentos }\end{array}$ & $\begin{array}{l}\text { Exostosis, } \\
\text { miositis } \\
\text { osificante, } \\
\text { calcificaciones } \\
\text { tendinosas, } \\
\text { quiste } \\
\text { poplíteo }\end{array}$ & & $\begin{array}{l}\text { Artritis reumatoide } \\
\text { crónica, tendinitis } \\
\text { crónica, } \\
\text { fibromialgia }\end{array}$ & $\begin{array}{l}\text { Osteoartritis } \\
\text { espondilartritis, } \\
\text { espondilosis }\end{array}$ & $\begin{array}{l}\text { Sarcoma, } \\
\text { condroma }\end{array}$ \\
\hline
\end{tabular}

especificidad hacia los diferentes tejidos afectados, como son músculos, huesos, tendones y ligamentos, vasos, nervios y tejidos blandos; y tropismo funcional, por todas las fases de la inflamación. La composición del medicamento actúa en sinergismo generando un terreno favorable para la actuación de los diferentes componentes del mismo, generando así una retroalimentación negativa, que hace viable la regresión de la enfermedad a estadios menos severos.

El medicamento biorregulador nos aporta una acción global sobre el cuadro clínico, con muy limitados efectos secundarios, y resultados clínicos comparables a los de tratamientos convencionales ${ }^{12-17}$.

El medicamento base para la OsteoArtrosis es el ZH (Tabla 3). Su composición nos asegura una actuación sobre todas las fases del deterio- ro articular (Figura 5), con niveles de eficacia comparables a medicamentos convencionales como AINEs y corticoides, in vivo, e in vitro, en los que "la suplementación de un medio de cultivo condrocitario que incluya suero bovino fetal con una solución inyectable de este medicamento al 10\%, mejora su rendimiento en un 20\% para el cultivo primario y un $25 \%$ en el secundario sin perjudicar al componente celular"12.

La concepción de la osteoartrosis como una insuficiencia del "órgano articular", al entender la articulación como una estructura compleja formada no solo por la cápsula articular, membrana sinovial, espacio articular y cartílago articular, sino por la estructura periarticular, el hueso subcondral, periostio, ligamentos y tendones, nervios y sistema miofascial 6 , así como el concepto de "facilitación muscular 
Tabla 3. Composición e indicaciones clínicas del Medicamento biorregulador ZH.

Cartilago suis (cartílago)

Artrosis deformante. Coxitis. Periartritis escapulohumeral.

Trastornos circulatorios.

Funiculus umbilicalis suis (cordón umbilical de Wharton Sulze)

Lesiones del tejido conjuntivo

Embryo suis (embrión)

Para la revitalización en fases celulares

Placenta suis (placenta)

Trastornos circulatorios. Sabañones

Toxicodendron quercifolium (hiedra venenosa) Notas:

Reumatismo y neuralgias, que empeoran en tiempo húmedo y

frío. Poliartritis. Enfermedades de las mucosas y del tejido conjuntivo.

\section{Arnica montana (árnica)}

Estimulación de la curación de heridas. Reumatismo. Dolores originados

por caídas, golpes o contusiones. Dolores en la espalda

y los miembros.

\section{Solanum dulcamara (dulcamara)}

Medicamento contra los efectos del tiempo húmedo. Sensación

de haberse enfriado. Afecciones reumáticas.

\section{Symphytum (consuelda)}

Traumatismos en tendones, ligamentos y periostio. Actúa en las articulaciones en general. Neuralgia de la rodilla.

Sanguinaria canadensis (sanguinaria del Canadá)

Dolores neurálgicos y reumáticos de articulaciones y músculos.

\section{Sulfur (azufre)}

Medicamento para favorecer la capacidad de reacción en todas las enfermedades crónicas. Favorece catalíticamente la actividad celular.

Nadidum (NAD) (nicotinamida adenina dinucleótido)

Coenzima. Estimulación de la oxidación en la cadena respiratoria.

\section{Coenzima A}

Coenzima para las transacetilaciones.

\section{Acidum a-liponicum (ácido tioctánico)}

Coenzima en la descomposición del ácido pirúvico.

Natrium oxalaceticum (oxalacetato sódico)

Factor activo del ciclo de Krebs y de los sistemas redox. Sensibilidad a la humedad y al viento.

\section{Acidum silicicum (ácido silícico)}

Debilidad y lesiones del tejido conjuntivo. Debilidad de los ligamentos. 
local" según el cual la presencia de estímulos aberrantes genera el aumento del umbral doloroso, nos dan una idea de la complejidad del problema y de la necesidad de una terapéutica que contemple todos los aspectos de la misma ${ }^{20}$.

\section{Protocolo de actuación}

Nuestra experiencia nos indica que debemos plantear dos objetivos terapéuticos, primero eliminar el dolor, la rigidez y el cuadro disfuncional que provocan la discapacidad y la limitación a la hora de instaurar tratamientos como los soportes plantares. Para ello, siempre que el paciente presente este cuadro neurogénico utilizamos el medicamento Bioregulador NRI (Tabla 4), en infiltración local terapéutica, utilizando la técnica convencional (Figura 6).

Para ello infiltramos periestructural a la $1^{\mathrm{a}}$ AMTF, en espacio intermetatarsal, utilizando agujas $30 \mathrm{G}$, con un volumen de $1,1 \mathrm{ml}$, y en cara dorso medial de la interlinea articular, un volumen de $0,5 \mathrm{ml}$. La sesión se repite un máximo de tres veces, a razón de una sesión por semana.

Y en segundo lugar, el tratamiento del deterioro articular en sí, tanto del cartílago como tal, de la sinovitis (si existiera) y del complejo articular, implicado en el proceso evolutivo de la OA. Si conseguimos la estabilización del área afectada, a nivel morfo-funcional y sensitivo, lograremos el retraso del deterioro articular, mejorando la movilidad y la percepción del

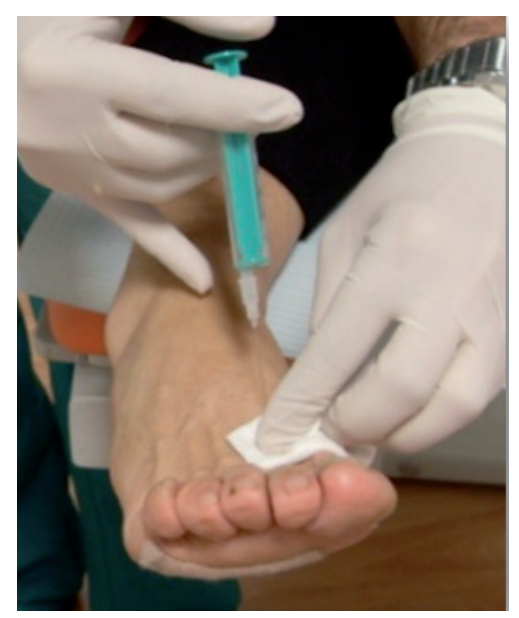

Figura 6. Infiltración en I espacio intermetatarsal para tratamiento de $\mathrm{OA}$. área afectada. Con este fin utilizamos el medicamento ZH (Tabla 3), por sus características organotrópicas y trópico funcionales, como comentamos anteriormente, en infiltración terapéutica periestructural, con soporte oral durante períodos largos de tratamiento, según la clínica y el grado de deterioro que presente cada paciente.

\section{CONCLUSIONES}

La OA es un síndrome de amplia presencia en la población, con mayor afectación en población anciana y en la mujer. El deterioro funcional y la limitación que genera provocan un elevado consumo de analgésicos y antiinflamatorios que no solucionan el cuadro clínico y sí provocan numerosos efectos secundarios, por lo que el paciente demanda tratamientos más resolutivos y mejor tolerables. El diagnóstico certero en estadíos iniciales de la patología y la comprensión del concepto de "órgano articular", mejorarán las perspectivas terapéuticas. La OA en las articulaciones de carga, particularmente en $1^{\circ}$ articulación metatarsofalángica del pie es altamente frecuente y motivo de gran cantidad de consultas dentro del ámbito podológico. La implicación de elementos periarticulares genera cuadros complejos en los que existe una alteración nos solo articular/funcional sino de carácter propioceptiva de muy difícil manejo clínico. La Medicina Biorreguladora nos aporta sustancias con organotropismo, hacia el tejido afectado y tropismo funcional sobre el proceso fisiopatológico, con una gran seguridad en el manejo clínico y muy limitados efectos secundarios. Las diferentes presentaciones nos ofrecen la posibilidad de tratamientos en la propia consulta y complementarlos con medicación, en comprimidos, gotas e incluso pomadas. La infiltración in situ de los biorreguladores aportará un efecto extra al aumentar el nivel de alerta del sistema inmunológico gracias a la punción local. 
Tabla 4. Observaciones farmacológicas y clínicas del medicamento Bioregulador NRI.

Causticum Hahnemanni (mezcla de bisulfato potásico incinerado e hidróxido cálcico)

Afecciones artríticas y reumáticas crónicas. Dolores desgarradores

y lancinantes de músculos y tendones.

Colchicum (cólquico)

Reumatismo muscular y articular. Dolor en la espalda. Agravación

especialmente en tiempo frío.

Colocynthis (coloquíntida)

Dolores punzantes y fulminantes, tanto en movimiento como en

reposo. Neuralgias, especialmente ciática. Sensación de tener

los músculos y tendones muy cortos.

Ferrum metallicum (hierro metálico)

Síndrome hombro-brazo (especialmente izquierdo). Lumbalgia.

Dolores en la cadera y en la tibia. Anemia secundaria. Migraña

Lithium benzoicum (benzoato de litio)

Diversos tipos de reumatismo y gota. Diátesis úrica

\section{Rhus toxicodendron (hiedra venenosa)}

Reumatismo y neuralgias que empeoran en tiempo húmedo, al mojarse por la lluvia y en reposo. Mejoría con tiempo cálido y seco

y con el movimiento como, por ejemplo, neuralgias del brazo

e intercostales. Ciática. Dolores en las extremidades.

\section{Spiraea ulmaria (ulmaria)}

Reumatismo muscular y articular. Epicondilitis (codo del tenista).

\section{Gnaphalium polycephalum (gnafalio)}

Ciática con parestesias y punzadas. Lumbalgia con sensación de

entumecimiento en la parte inferior de la espalda.

\section{BIBLIOGRAFÍA}

1. Yukihisa Fukudaa, Shinro Takaia, Nobuyuki Yoshinoa, Kouhei Muraseb, Sadami Tsutsumic, Ken Ikeuchid,Yasusuke Hirasawaa. Impact load transmission of the knee joint-influence of leg alignment and the role of meniscus and articular cartilage. Clinical Biomechanics.2000; 15 (7): 516-521.

2. Radin EL, PaulIL. Response of joint to impact loading, I: in vitro wear. Arthr Rheum, 1971;14:356-62.

3. OConnor JJ, Jhonson I. transmission of rapidly applied load though articular cartilage: the mechanics of osteoarthrosis. Turner-Smith AR, ed. Micromovement in Orthopaedics. Oxford: Clarendon Press; 1993: 244-268.

4. Bonnet CS, Walsh DA. Osteoarthritis: angiogenesis and inflammation. Rheumatology 2005;44:7-16.

5. Yasuda T, Poole AR. A fibronectin fragment induces type II collagen degradation by colagenase through an interleukin-1-mediated pathway. Arthritis Rheum 2002; 46: 138-48.

6. Quintero, Monfort, Mitrovic. Osteoartrosis. Biología, Fisiología, clínica y tratamiento. Ed Panamericana 2010: 280.

7. Pelletier JP, Martel-Pelletier J, Abramson SB. Osteoarthritis, an inflammatory Disease. Potencial implication for the selection of new therapeutic targets. Arthritis\&Rheumatism 2001;44 (6):1237-1247.

8. Walsh D. Angiogenesis and arthritis. Rheumatology 1999;38:103-12. 
9. Weinstein SL, Buckwalter JA. Turek's Orthopaedics; Principles and their application. Rheumatic Diseases: Diagnosis and management. JB Lippincott Company $5^{\text {th }}$ ed. 1984: 151-60.

10. Ekcsterin F, Burstain D, Link TM. Quantitative MRI of cartilage and bone: degenerative changes in OA. NMR Biomed. 2006;19: 822-54.

11. Alta Smit. Tratamiento de las enfermedades músculoesqueléticas. Journal of Biological Medicine.2004; 2: 53-60.

12. García-Mediavilla MV, Iglesias Muñoz FJ, Gudiña Pérez EJ, Hernández González LC,1 del Valle Soto M, Banco de Tejidos Humanos de la Fundación Clínica San Francisco de León. INBIOTEC, Universidad de León. Departamento de Morfología y BiologíaCelular. Escuela de Medicina del Deporte, Universidad de Oviedo. La respuesta de condrocitos articulares cultivados in vitro bajo los efectos de Zeel T. Medicina Biológica 2004; 3: 98-104.

13. Birnesser H, Klein P, Weiser M. Tratamiento de la gonartrosis. Un medicamento homeopático con la misma eficacia que los inhibidores de la COX-2. Med Biol 2005; 2:52-58.

14. Weiser M, Metelmann H. Tratamiento de la gonartrosis con Zeel T solución inyectable. Resultados de un estudio de aplicación. Med Biol 1996; 1:141-9.

15. Orlandini A, Rossi M, Setti M. La eficacia de Zeel T y nuevos métodos de investigación en reumatología. Med Biol 1998; 1:139-40.

16. Gottwald R, Weiser M. Tratamiento de la gonartrosis con Zeel T. Med Biol 2000; 4: 109-113.

17. Wodick RE, Steininger K, Zenner S. Tratamiento de las artropatías degenerativas con Zeel pomada. Resultados de un estudio de aplicación en 498 pacientes. Med Biol 1997; 2:44-51.

18. Antonio Jurado Bueno, Iván Medina Porqueres. Tendón, valoración y tratamiento en fisioterapia. Ed Paidotribo 2008:531.

19. Jan Kersschot. Biopunture, a clinic guide. Inspiration Publishing 2008: 15.

20. Leon Chaitow, Judith Walter DeLany. Aplicación clínica de las técnicas neuromusculares. Editorial Paidotribo 2006: 70-74. 